http://jmscr.igmpublication.org/home/ ISSN (e)-2347-176x ISSN (p) 2455-0450 crossref DOI: https://dx.doi.org/10.18535/jmscr/v9i10.13

\author{
Dournal Of Medical Science And Clinical Research \\ IGM Publication \\ An official Publication of IGM Publication
}

\title{
SKINTED: A New Type of Eczematous Dermatitis
}

\author{
Authors \\ Dr Ashwani Rana ${ }^{1}$, Dr Prajul Mehta ${ }^{2 *}$ \\ ${ }^{1}$ MD Dermatology CH Joginder-nagar, Mandi, H.P. India \\ ${ }^{2}$ MD Dermatology CH Theog, Shimla, H.P. India \\ *Corresponding Author \\ Dr Prajul Mehta \\ CH Theog, Shimla, H.P. India
}

\begin{abstract}
We hereby report a case of bilateral total knee replacement surgery, developing eczematous lesions around the knee surgery area on both sides several months after surgery. The denervation injury due to dermal nerve transections may lead to autonomic disturbance in the involved area and contribute to the development of dermatitis.
\end{abstract}

\section{Introduction}

The term "surgery of the knee, injury to the infrapatellar branch of the saphenous nerve, traumatic eczematous dermatitis" SKINTED was initially used by Verma and Mody. ${ }^{1}$

"Neuropathy dermatitis" was proposed by Sharquie et al., ${ }^{[2]}$ wherein they reported eruptions similar to those observed by Verma and Mody. Surgery of the knee, injury to the infrapatellar branch of the saphenous nerve, traumatic eczematous dermatitis (SKINTED) is an autonomic denervation dermatitis specific to total knee replacement surgery occurring around the healed surgical scar area.

\section{Case Report}

A 68 year old male presented in our outpatient department of dermatology with a history of bilateral total knee replacement surgery 9 months ago presented with itchy, oozy, red raised lesions over the left knee and raised lesions over the right knee. (Figure 1)

The patient had an uneventful postoperative period with well functioning knees at the time of presentation of skin lesions. There was no history of the use of any topical agent/contact allergen during the preoperative and postoperative periods. There was no prior history of metal allergy or other allergic conditions, diabetic neuropathy, chronic liver, and renal condition. There were no skin lesions in any other part of the body. Gram stain and fungal examination (potassium hydroxide) did not reveal any organism. Laboratory tests including complete cell blood count, kidney and liver function tests, and total serum IgE levels were within normal limits. There were no signs and symptoms of venous stasis; therefore, vascular duplex scan was not done.

The cutaneous examination revealed erythematous eczematous plaque lateral to midline healed scar 
present on bilateral lower leg; more prominent over the left side.

The mid-potent topical corticosteroid once daily, oral antihistamine for pruritis daily and application of bland emollients was prescribed; the patient improved in 2 weeks and was advised daily good moisturizer over the affected area thereafter resulting in clinical improvement.

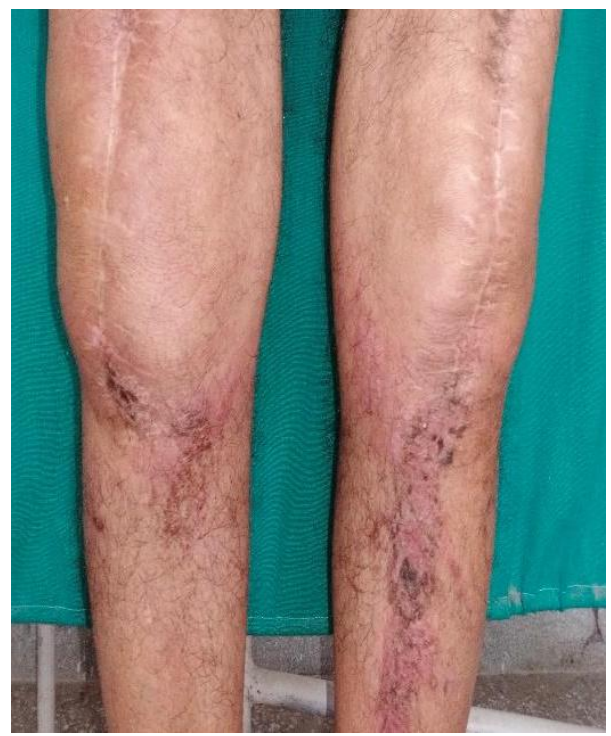

Figure 1 Bilateral Erythematous Eczematous plaque lateral to midline scar

\section{Discussion}

The infrapatellar branch of the saphenous nerve (IPBSN) is a purely sensory nerve that crosses from medial to lateral in the infrapatellar area, supplying cutaneous sensation to the inferolateral knee area. Variations of its course and the branching pattern have been described in various anatomical studies. ${ }^{3,4}$ Iatrogenic injury of the IPBSN has been described within orthopaedic literature during several knee surgical procedures, including TKA. The IPBSN is most often resected during total knee replacement surgery using median parapatellar incision. Infrapatellar nerve damage causes hypoesthesia and cutaneous eruption. The cutaneous autonomic nervous system plays a crucial part in regulating sweat gland function, vasomotor activity, and skin blood flow. Skin barrier function is a result of complex and poorly understood processes and normal functioning of sweat glands, sebaceous glands, and cutaneous microcirculation (which in turn depends on autonomic nerve activity) appears to be necessary for maintenance of the skin barrier. ${ }^{5}$ In addition, acetylcholine and catecholamines secreted from autonomic nerve endings are thought to play a role in keratinocyte proliferation, adhesion, migration, and differentiation - .another instance of the role of autonomic nerve activity in skin barrier homeostasis. ${ }^{6}$

Verma and Mody ${ }^{[1]}$ in 2009 postulated that damage to the cutaneous nerve during surgery alters the barrier function of the epidermis, resulting in transepidermal water loss, causing xerosis, and the eventual development of an eczematous dermatitis.

SKINTED is a subset of proposed clinical term - Autonomic denervation dermatitis (ADD). ${ }^{7}$

"SKINTED" is a site- . And procedure- specific diagnosis while the term ADD would be applicable to all eczematous eruptions at or around surgical sites irrespective of site and nature of the operative procedure.

\section{Declaration of Patient Consent}

The authors certify that they have obtained all appropriate patient consent forms. In the form the patient (s) has/have given his/her/ their consent for his/her/their images and other clinical information to be reported in the journal. The patients understand that their names and initials will not be published and due efforts will be made to conceal their identity, but anonymity cannot be guaranteed.

\section{Conflict of Interest- Nil}

\section{References}

1. Verma SB, Mody BS. Explaining a hitherto nameless condition: 'SKINTED'. Clin Exp Dermatol 2009; 34: e465-e466.

2. Sharquie KE, Noaimi AA, Alaboudi AS. Neuropathy dermatitis following surgical nerve injury. Case Rep Dermatol Med 2011;2011:234185. 
3. Kartus J, Ejerhed L, Eriksson BI, Karlsson J. The localization of the infrapatellar nerves in the anterior knee region with special emphasis on central third patellar tendon harvest: a dissection study on cadaver and amputated specimens. Arthroscopy 1999;15(6):577.

4. Ebraheim NA, Mikhail AO. The infrapatellar branch of the sapenous nerve: an anatomical study. J Orthop Trauma 1997; 11: 195-199.

5. Nomura T, Yoshida-Amano Y, Yoshida K, Fujii A, Tanahashi M, Sugiyama Y, et al. Relationships between transepidermal water loss, cutaneous microcirculatory function and autonomic nervous activity. Int J Cosmet Sci 2017;39:275-83

6. Roosterman D, Goerge T, Schneider SW, Bunnett NW, Steinhoff M. Neuronal control of skin function: The skin as a neuroimmunoendocrine organ. Physiol Rev 2006;86:1309- 79

7. Madke BS, Mhatre MA, Kumar P, Singh AL, Patki AH. Autonomic denervation dermatitis: a new type of eczematous dermatitis. Clin Dermatol Rev 2017;1: 61. 